\title{
On the design of tone-free modulators
}

\author{
Risbo, Lars
}

Published in:

IEEE transactions on circuits and systems - 2, Analog and digital signal processing

Link to article, DOI:

$10.1109 / 82.363540$

Publication date:

1995

Document Version

Publisher's PDF, also known as Version of record

Link back to DTU Orbit

Citation (APA):

Risbo, L. (1995). On the design of tone-free modulators. IEEE transactions on circuits and systems - 2, Analog and digital signal processing, 42(1), 52-55. https://doi.org/10.1109/82.363540

\section{General rights}

Copyright and moral rights for the publications made accessible in the public portal are retained by the authors and/or other copyright owners and it is a condition of accessing publications that users recognise and abide by the legal requirements associated with these rights.

- Users may download and print one copy of any publication from the public portal for the purpose of private study or research.

- You may not further distribute the material or use it for any profit-making activity or commercial gain

- You may freely distribute the URL identifying the publication in the public portal

If you believe that this document breaches copyright please contact us providing details, and we will remove access to the work immediately and investigate your claim. 
[10] J. Canaris, "A VLSI architecture for the real time computation of discrete trigonometric transforms," J. VLSI Signal Processing, vol. 5, no. 1, pp. 95-104, 1993.

[11] Y. H. Chan and W. C. Siu, "On the realization of DCT using the distributed arithmetic," IEEE Trans. Circuits Syst.-1, vol. 39, no. 9, pp. 705-712, 1992.

[12] B. G. Lee, "Input and output index mappings for a prime-factordecomposed computation of discrete cosine transform," IEEE Trans Acoust., Speech. Signal Process., vol. 37, no. 2, pp. 237-244, 1989.

\section{On the Design of Tone-Free $\Sigma \Delta$ Modulators}

\author{
Lars Risbo
}

\begin{abstract}
Traditional one-bit $\Sigma \Delta$ modulators used for $A / D$ and $D / A$ conversion produce very predominant tones near half the sample rate which might intermodulate in the analog converter section and cause inband tones. This paper demonstrates how the use of chaos can substitute dither as a means for extinguishing these tones. Especially, modulator feedback filters derived from noise transfer functions having an all-pass term seem very promising.
\end{abstract}

Index Terms-Sigma-Delta modulation, tones, chaos, dithering.

\section{INTRODUCTION}

$\Sigma \Delta$ modulators are extensively used in the design of oversampling audio A/D and D/A data converters [1],[2].

One of drawbacks the of conventional $\Sigma \Delta$ modulators is the possible existence of tones in the output spectrum [3]-[7]. A very common property is that when the modulator is fed with a dc-input, $\lambda$, the modulator spectrum contains a very strong out-of-band tone at the frequency $(1-\lambda) f_{s} / 2$, where $f_{s}$ is the sample rate [2], [3], [6]. Simulations have indicated that the tone close to $f_{s} / 2$ causes the in-band noise power to vary periodically with the frequency $\lambda f_{s}$ [5]. Such periodic noise modulation is not observable on usual power spectra estimates but can be seen in the time domain and might be perceived as a tone by the human ear [3]. The frequency modulation of the tone near $f_{s} / 2$ seems to be very general, i.e., sinusoidal input with frequency $f$ produces characteristic sideband tones at the frequencies $f_{s} / 2-k f$ for integer $k$ [2]. In addition, the high-frequency tone and the sidebands can produce in-band intermodulation components produced by analog circuit nonlinearities [8], [9].

The conclusion from the observations mentioned above seems to be that precautions against the out-of-band tones must be taken if $\Sigma \Delta$ converters free from audible tonality are to be designed.

Enhanced suppression of both in-band noise and in-band tones can generally be achieved by increasing the order of the modulator, since this normally allows a higher attainable in-band loop gain. However, this has very little or no effect on the amplitude of the tones near

Manuscript received January 11, 1994; revised, July 16, 1994. This paper was recommended by Associate Editor Sayfe Kiaei.

The author is with the Electronics Institute, Technical University of Denmark, DK-2800 Lyngby, Denmark.

IEEE Log Number 9407420.

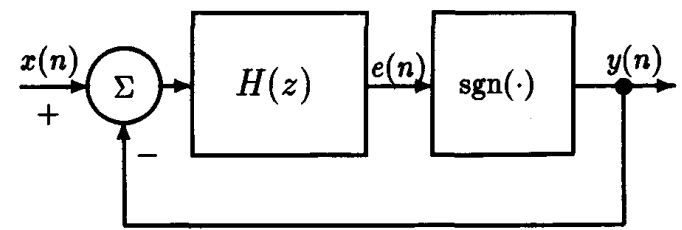

Fig. 1. $\Sigma \Delta$ modulator with feedback filter $H(z)$.

$f_{s} / 2$ since the out-of-band loop gain must remain nearly the same in order to preserve loop stability. Even eighth-order modulators seem to suffer from these high-frequency tones [2].

A different approach to tone suppression is the introduction of a degree of 'uncertainty' into the modulation. This is accomplished either by adding dither noise into the modulator loop [3]; [4] or by the use of a chaotic modulator [4], [6], [7]. The purpose of this letter is to compare these two strategies with respect to the suppression of the tones near $f_{s} / 2$.

\section{FEEDBACK FILTER DESIGN FROM NTF-PROTOTYPES}

Fig. 1 shows a typical $\Sigma \Delta$ modulator composed of a feedback filter (i.e., loop filter) $H(z)$ and a one-bit quantizer (i.e., signum function).

When the one-bit quantizer is crudely modeled as a unity gain with an additive noise source, the noise transfer function, $\operatorname{NTF}(z)$, from the noise source to the modulator output is given by:

$$
\operatorname{NTF}(z)=\frac{1}{1+H(z)}
$$

The NTF describes how the quantization noise is spectrally shaped and suppressed in the base-band.

$\Sigma \Delta$ modulators are typically designed by specification of a desired high-pass noise transfer function, i.e., an NTF-prototype [1], [5], [10], [11]. From an NTF-prototype given as a rational transfer function NTF $(z)=A(z) / B(z)$ the feedback filter $H(z)$ can be derived using (1):

$$
H(z)=\frac{1}{\operatorname{NTF}(z)}-1=\frac{B(z)-A(z)}{A(z)}
$$

Since delay free loops around a quantizer are not implementable, $H(z)$ must have at least a one sample delay, i.e., the associated impulse response must have the property $h(k)=0, k<1$ [1], [4]. Equation (2) shows that this is achieved when the NTF-prototype is scaled such that $A(z)$ and $B(z)$ have the same highest-order $z$-term. This necessary scaling causes a unity passband gain NTF-prototype to have a higher passband gain and a lower stop-band attenuation after scaling.

A good in-band noise suppression can be obtained by designing the NTF-prototype as a Chebychev II high-pass filter with unit circle zeros distributed in the base-band [1] (see also [11] for optimum unit circle zero locations). Such scaled prototype filters can easily be designed using the MATLAB Signal Processing Toolbox commands [5], [12]:

$$
\begin{aligned}
{[\mathrm{A}, \mathrm{B}] } & =\operatorname{cheby} 2\left(\mathrm{~N}, \mathrm{Rs}, \mathrm{fb},{ }^{\prime} \text { high }^{\prime}\right) \\
\mathrm{A} & =\mathrm{A} / \mathrm{A}(1)
\end{aligned}
$$


where $N$ is the filter order, Rs is the desired base-band attenuation in $\mathrm{dB}$ (prior to scaling) and $f b$ is the upper base-band edge normalized with respect to $f_{s} / 2$.

Increasing Rs will obviously improve the suppression of inband noise and thereby the SNR; however, the necessary scaling causes the high-frequency gain of the NTF-prototype to increase and, unfortunately, too much high-frequency noise destabilizes the modulator loop, i.e., the stable amplitude range of the modulator is reduced [1], [10], [11]. This phenomenon is caused by an overload of the simple one-bit quantizer, i.e., the quantizer output power is always unity and must be shared between the in-band signal and the circulating noise.

The feedback filter design is thus a trade-off between in-band noise suppression performance and loop stability. See [5], [10], [11] for a discussion of design trade-offs and stability criteria for high-order modulators.

\section{Chaotic $\Sigma \Delta$ Modulators}

An $N$ th-order $\Sigma \Delta$ modulator is a nonlinear discrete-time dynamical system, which can be analyzed by observing trajectories in a corresponding $N$-dimensional state-space. Generally, the state-space has a large number of periodic trajectories (i.e., limit cycles) with different periods [13].

It can be shown that if all poles of the feedback filter $H(z)$ are inside the unit circle, the limit cycles have attracting regions in the state-space which cause the modulator to lock asymptotically into strictly periodic modes [13].

If one or more of the $H(z)$ poles are outside the unit circle, the limit cycles will be unstable, i.e., even small perturbations in the state space will be amplified exponentially in time [6]. The sensitivity to initial conditions and a high density of limit cycles are important characteristics of chaos. Chaotic systems are unpredictable on long time scales and generate nonperiodic outputs. Despite these properties, chaos does generally not guarantee that the quantization noise of $\Sigma \Delta$ modulators is free from tones [6], [7].

The moduli of the poles outside the unit circle is a measure of the 'amount of chaos' in the modulator, i.e., how fast nearby points in state-space diverge per time step. The base two logarithms of the pole moduli are in fact equal to the so-called Lyapunov exponents used in chaos theory [13] and these exponents express the loss of state-space information in bits per time step.

\section{NTF-PRototypes with ALL-PASS TeRms}

In order to obtain chaos, $H(z)$ must have at least one pole outside the unit circle. This is achieved when an NTF-prototype with zeros outside the unit circle is used, cf. (2).

An initial approach was to use a standard $\Sigma \Delta$ modulator and scale up the $H(z)$ unit circle poles with a certain factor; however this procedure reduces the in-band noise suppression significantly due to the resulting lower loop gain [6], [13].

Another way to obtain chaos is to use a minimum phase NTFprototype and reflect one or more zeros to their reciprocal locations outside the unit circle, as demonstrated for FIR filters in [4]. The necessary scaling of the zero-reflected prototype scales up the magnitude characteristic by the product of the moduli of the reflected zeros, and this reduces the loop stability considerably. The stability was secured by adding more quantizer levels in [4]. The benefit of zero-reflection is that the in-band noise suppression is only reduced proportional to the necessary scaling.

An alternative to zero-reflection is to introduce an all-pass term into a standard IIR NTF-prototype with all zeros on the unit circle. A first-order all-pass term must naturally have a (real valued) zero outside the unit circle and a pole inside the unit circle which is the reciprocal of the zero [14]. The advantages of an all-pass extended NTF-prototype are that the zeros are preserved on the unit circle for optimum in-band noise suppression and that the degree of chaos can be adjusted independently of the NTF magnitude characteristic by adjusting the all-pass zero location. A normalized all-pass term with a zero at $z=\alpha,|\alpha|>1$ has the form:

$$
\mathrm{NTF}_{\mathrm{ap}}(z)=\frac{1-\alpha z^{-1}}{1-\frac{\alpha}{|\alpha|^{2}} z^{-1}}
$$

This all-pass term may be multiplied on any normalized NTFprototype thus giving a new normalized NTF-prototype with a filter order increased by one. Since the magnitude response of (3) is equal to $|\alpha|$, the loop stability deteriorates as $|\alpha|$ is increased. Consequently, 'more chaos' and thereby better tone suppression can be obtained at the expense of a lower stable amplitude range.

\section{Design and Simulation Examples}

In this section, three methods for suppressing the tones near $f_{s} / 2$ are compared, i.e., NTF-prototype zero scaling, one-bit quantizer dithering, and finally, the use of an all-pass extended NTF-prototype.

For the sake of comparisons, the simulation examples will all be for sixth-order modulators based on Chebychev II prototypes designed with MATLAB. All modulators are intended for 64 times oversampling, i.e., $\mathrm{fb}=1 / 64 \cdot 20 / 22.05$ (suitable for e.g., $20 \mathrm{kHz}$ base-band and $64 \cdot 44.1 \mathrm{kHz}$ sample rate). An additional design criterion was to ensure a stable input range up to 0.35 relative to full scale. The simulations use a dc-input of 1/256 corresponding to possible high-frequency tones that might intermodulate and generate base-band tones at $f=1 / 128 \cdot f_{s} / 2$. The spectra shown in this section are obtained by averaging Kaiser-Bessel windowed 8 k FFT power spectra for sequences of two mill. one-bit samples. The heavy averaging allows tones to be distinguished from random noise. In this context, the designation 'tone free' is used when possible tones are comparable to or below the noise floor provided by the given spectral resolution. An enhanced spectral resolution (e.g., larger FFT size) can naturally reveal more tones because the noise floor is lowered. For each spectrum the in-band noise power is computed by summing up the power of the appropriate FFT-bins.

The first modulator tested had a prototype designed with the parameters $N=6$ and $\mathrm{Rs}=120 \mathrm{~dB}$. This modulator is stable for input amplitudes up to approximately 0.35 relative to full scale. Fig. 2 shows the simulated output power spectrum. The in-band noise power is approximately $-142 \mathrm{~dB}$ (relative to full scale) corresponding to more than 21 bits of resolution. However, the tone seen near $f_{s} / 2$ is no more than $6.7 \mathrm{~dB}$ below full scale- the tone is in fact stronger than any possible modulator input!

Experiments with scaling of the NTF-prototype zeros were then carried out. This resulted in a design based on a prototype with $N=$ 6 and Rs $=90 \mathrm{~dB}$. A zero scaling by a factor of 1.06 was necessary to suppress the high-frequency tones below the noise floor. The resulting tone free spectrum is shown on Fig. 3. The in-band noise power is as high as $-61 \mathrm{~dB}$ and this is a consequence of the NTF-zeros being removed from the unit circle. Furthermore, the experiments showed that this modulator is too unstable for any practical purposes.

The next approach was to add a dither noise source to the quantizer input. Obviously, this introduces more noise into the loop which consequently becomes more unstable. The NTF-prototype must therefore be designed with a somewhat lower Rs such that the same stable input range is preserved when dither is applied. The combination of $\mathrm{N}=6, \mathrm{Rs}=95 \mathrm{~dB}$ and a spectral white dither source with a uniform amplitude density from -0.5 to 0.5 resulted in a tone free design with a stable input range up to approximately 


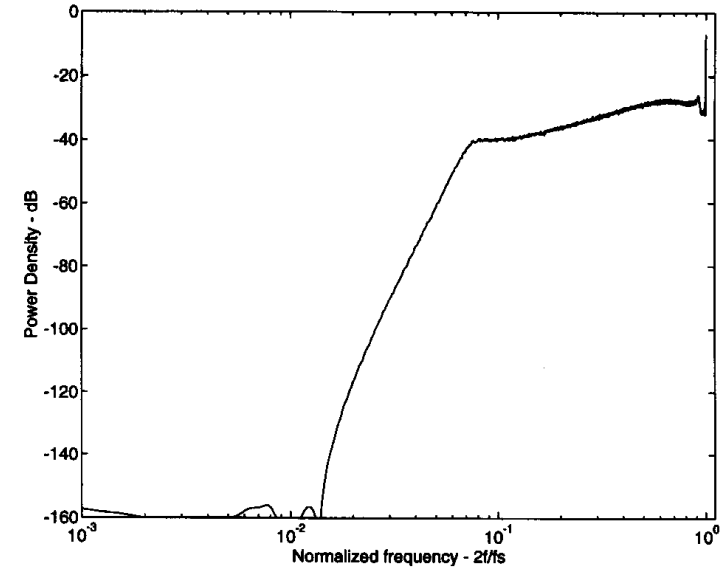

Fig. 2. Power spectrum from a simulation of a sixth-order modulator with Rs $=120 \mathrm{~dB}$.

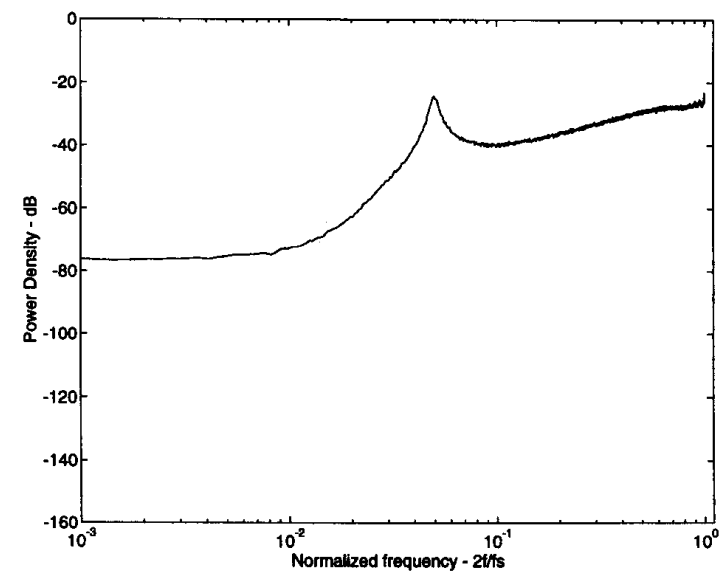

Fig. 3. Power spectrum from a simulation of a sixth-order modulator with $\mathrm{Rs}=90 \mathrm{~dB}$ and an NTF-zero scaling of 1.06 .

0.35. The spectrum is showed in Fig. 4 and the in-band noise power is approximately $-115 \mathrm{~dB}$. A peak at $-26.4 \mathrm{~dB}$ is seen at $(1-1 / 256) f_{s} / 2$. When a lower dither span is applied, both a higher SNR and stable input range is obtained; however, the high-frequency tone becomes stronger.

Finally, a sixth-order modulator was obtained from a fifth-order NTF-prototype extended by a first-order all-pass term, cf. (3). Experiments have shown that negative values of $\alpha$ are far more efficient than positive values in respect to suppression of high-frequency tones-probably because an NTF-zero near $z=-1$ corresponds to $f=f_{s} / 2$. The combination of $\mathrm{N}=5, \mathrm{Rs}=80 \mathrm{~dB}$ and $\alpha=-1.25$ resulted in a tone free modulator with a stable input range up to approximately 0.35 . The spectrum is showed in Fig. 5 and the inband noise power is approximately $-105 \mathrm{~dB}$. A peak at $-37.9 \mathrm{~dB}$ is seen at $(1-1 / 256) f_{s} / 2$. The modulator has obviously a fifth-order noise shaping amplitude characteristic and this is limiting the SNR. If the total order is increased to 7 , it is possible to obtain a tone free design with a better SNR and the same stable amplitude range: the parameters $N=6, \mathrm{Rs}=92 \mathrm{~dB}$ and $\alpha=-1.2$ yields an in-

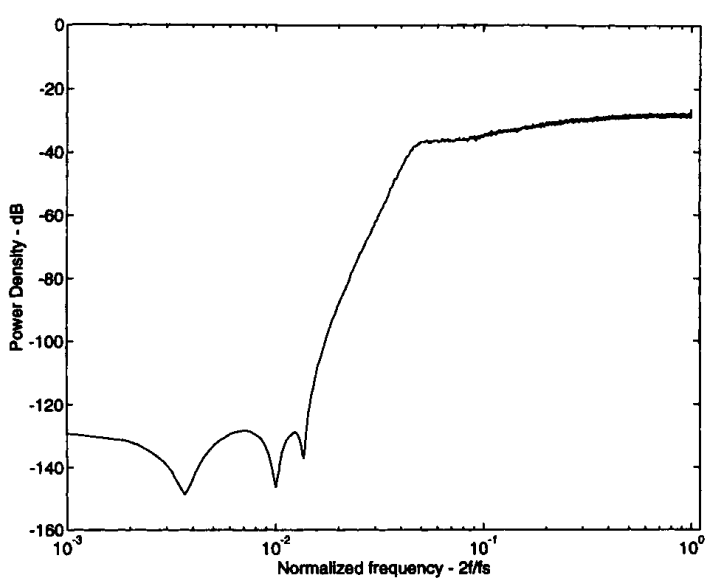

Fig. 4. Power spectrum from a simulation of a sixth-order modulator with $\mathrm{Rs}=95 \mathrm{~dB}$ using dither uniformly distributed over the interval $[-0.5,0.5]$.

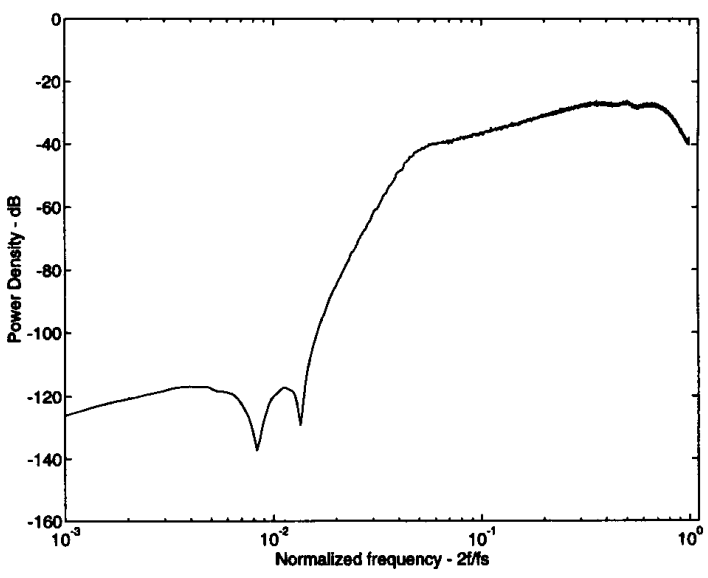

Fig. 5. Power spectrum from a simulation of a fifth-order modulator with $\mathrm{Rs}=80 \mathrm{~dB}$ extended to sixth-order using an all-pass term with $\alpha=-1.25$.

band noise power of approximately $-114 \mathrm{~dB}$, i.e., almost the same performance as for the dithered sixth-order example.

\section{CONCluSion}

It has been demonstrated that the use of chaos effectively can substitute dither as a means for suppression of the predominant tones near half the sample rate in one-bit $\Sigma \Delta$ modulators. In particular, the use of a general all-pass term seems to be very advantageous. Investigations have shown that a first-order all-pass term with a real valued negative zero outside the unit circle is a feasible choice. The price paid for the tone suppression is that both dithering and the use of chaos reduce the loop stability significantly. In order to restore stability, the in-band loop gain must be reduced resulting in a lower attainable SNR for a given modulator order. However, this can to some extend be remedied by increasing the modulator order.

The presented examples indicate that the dithered modulator obtains a $9 \mathrm{~dB}$ better SNR than a chaotic modulator for the same total order and stable amplitude range. However, this is to some extend counterbalanced by the high-frequency tone being approximately 11 
$\mathrm{dB}$ weaker for the chaotic modulator example than for the dithered. Future research should therefore attempt to uncover the exact relations between tone strength, dither span and the all-pass zero modulus.

\section{REFERENCES}

[1] R. W. Adams et al., "Theory and practical implementation of a 5th-order Sigma-Delta A/D converter," J. Audio Eng. Soc., vol. 39, pp. 515-528, July/Aug. 1991.

[2] L. Risbo, "FPGA based 32 times oversampling 8th order Sigma-Delta audio DAC," in Proc. 96th AES Conv., preprint \# 3808, Feb. 1994.

[3] S. R. Norsworthy and D. A. Rich, "Idle channel tones and dithering in Delta-Sigma modulators," in Proc. 95th AES Conv., preprint \# 3711, Oct. 1993.

[4] S. R. Norsworthy, "Optimal nonrecursive noise shaping filters for oversampling data converters," in IEEE Proc. ISCAS '93, Chicago, IL, USA, May 1993, pp. 1353-1356.

[5] L. Risbo, " $\Sigma-\Delta$ Modulators-Stability Analysis and Optimization," Ph.D. dissertation, Electronics Institute, Technical University of Denmark, June 1994

[6] R. Schreier, "Destabilizing limit cycles in Delta-Sigma modulators with chaos," in IEEE Proc. ISCAS '93, Chicago, IL USA, May 1993, pp. 1369-1372

[7] M. Motamed, A. Zakhor, and S. Sanders, "Tones, saturation and SNR in double loop $\Sigma-\Delta$ modulators," in Proc. IEEE ISCAS '93, Chicago, IL USA, May 1993, pp. 1345-1348.

[8] S. Harris, "How to achieve optimum performance from Delta-Sigma A/D and D/A converters," in Proc. 93rd AES Convention, preprint \# 3417, Oct. 1992.

[9] R. W. Adams, private communication, Nov. 1992.

[10] L. Risbo, "Stability predictions for high-order Sigma-Delta modulators based on quasilinear modeling," in Proc. IEEE ISCAS '94, London, UK, May 1994, pp. 5.361-5.364.

[11] R. Schreier, "An empirical study of high-order single-bit delta-sigma modulators," IEEE Trans. Circuits Syst. II, vol. 40, no. 8, pp. 461-466, Aug. 1993.

[12] J. N. Little and L. Shure, Signal Processing TOOLBOX-User's Guide MathWorks, 1993.

[13] S. Hein and A. Zakhor, Sigma-Delta Modulators-Nonlinear Decoding Algorithms and Stability Analysis Norwell, MA: Kluwer Academic, 1993.

[14] A. V. Oppenheim and R. W. Schafer, Discrete-Time Signal Processing. Englewood Cliffs, NJ: Prentice Hall, 1989.

\section{On the Robust Design of $k$-Winners-Take-All Networks}

$$
\text { R. Perfetti }
$$

\footnotetext{
Abstract-A design methodology of $k$-Winners-Take-All (k-WTA) networks is proposed. The design is optimal in the sense that the allowed relative deviation from the nominal parameter values is maximized, while preserving a correct $k$-WTA behavior. Accuracy requirements do not limit the network size but only the number of winners.
}

\section{INTRODUCTION}

Networks realizing the Winner-Take-All (WTA) function have been extensively discussed in the recent literature (e.g., [1]-[4]). A k-WTA network is a competitive dynamical system which is able to select

Manuscript received February 28, 1994; revised July 21, 1994. This paper was recommended by Associate Editor S. Kiaei.

The author is with the Institute of Electronics, University of Perugia, I06100 Perugia, Italy.

IEEE Log Number 9407424. the $k$ larger, among $n$ input signals $(k<n)$. This task is performed by mutual inhibition among the $n$ processing units composing the network. This type of network has an obvious field of application in classification and pattern recognition problems [5].

Much of this work has been motivated by [6], where the authors apply the robust design methodology, proposed in [7], to the WTA network. To be more precise, the design problem investigated in [6] can be stated as follows: Find the parameters of a 1-WTA network such that the allowed deviation from the nominal values, corresponding to correct 1-WTA behavior, is maximum. The system model adopted in [6] gives a maximum allowed deviation which is a monotonically decreasing function of the number $n$ of units. The required accuracy increases so rapidly with $n$ that, in practice, the number of units is limited to at most $n=10$. In the present paper a slightly modified WTA model is proposed, requiring a relative accuracy which results to be independent of $n$. Since accuracy does not limit the network size, the more general robust design of a $k$ WTA network is taken into consideration. It is shown that accuracy requirements limit the number of 'winners' to at most $k=20$, if a precision of $1 \%$ is assumed.

\section{Design Methodology}

For sake of clarity most of the terminology is borrowed from [6]. The network architecture is composed of $n$ identical cells. The cell equation is:

$$
\frac{d x^{c}}{d t}=-x^{c}+\alpha \sum_{d \in C G} y^{d}+(\delta+1) y^{c}+\theta
$$

where $c \in C G=\{1 \cdots n\}$. The equation is identical for all cells ${ }^{1}$.

The sum over the cell grid $C G$ can be realized by a global summer [6]. So, this model is compatible with the simplified architecture proposed by Lazzaro et al. [1], allowing the number of links to increase only linearly with $n$. The network is inputless and it processes only the information represented by its initial state ${ }^{2}$. The nonlinear output function is given by:

$$
y^{c}=f\left(x^{c}\right)= \begin{cases}+1, & \text { if } x^{c} \geq+1 \\ x^{c}, & \text { if } 0<x^{c}<+1 \\ 0, & \text { if } x^{c} \leq 0\end{cases}
$$

Note that the nonlinear function (2) is the only structural difference between the present model and that adopted in [6]. This nonlinearity gives design constraints which do not depend on $n$. As a consequence, the accuracy requirements are independent on $n$, as it will be shown in Section III

Consider the $n$-dimensional network described by (1). We call it a $k$-WTA network if it satisfies the following two conditions:

1) For all initial states ${ }^{3}$, it converges to a final state consisting of exactly $k$ cells with maximum output value $(+1)$, and $n-k$ cells with minimum output value $(0)$.

2) The cells with maximum output value are precisely those with the larger initial states.

${ }^{1}$ The symbol $\theta$, instead of $k$, has been used for the threshold to avoid confusion with the number of winners.

${ }^{2}$ Some authors call inputs the thresholds; this definition is not appropriate since the threshold values are fixed a priori and do not carry any information.

${ }^{3}$ The only permissible exceptions are when there are identical initial conditions for two or more cells. Since these initial states have no practical interest they will be ignored in the following. 